PROCEEDINGS OF THE

AMERICAN MATHEMATICAL SOCIETY

Volume 134, Number 8, Pages 2309-2316

S 0002-9939(06)08545-5

Article electronically published on March 20, 2006

\title{
A NOTE ON SHARP 1-DIMENSIONAL POINCARÉ INEQUALITIES
}

\author{
SENG-KEE CHUA AND RICHARD L. WHEEDEN
}

(Communicated by Michael C. Lacey)

\begin{abstract}
Let $1<p<\infty$ and $-\infty<a<b<\infty$. We show by using elementary methods that the best constant $C$ (necessarily independent of $a$ and $b$ ) for which the 1-dimensional Poincaré inequality
\end{abstract}

$$
\left\|f-f_{a v}\right\|_{L^{1}[a, b]} \leq C(b-a)^{2-\frac{1}{p}}\left\|f^{\prime}\right\|_{L^{p}[a, b]}
$$

holds for all Lipschitz continuous functions $f$, with $f_{a v}=\int_{a}^{b} f /(b-a)$, is

$$
C=\frac{1}{2}\left(1+p^{\prime}\right)^{-1 / p^{\prime}}
$$

Let $B$ be a ball in $n$-dimensional Euclidean space $\mathbb{R}^{n}$, and let $1 \leq p, q<\infty$. Also, assume that $q \leq \frac{p n}{n-p}$ when $p<n$. Let $\operatorname{Lip}(B)$ denote the class of Lipschitz continuous functions on $B$, and let $\operatorname{Lip}_{0}(B)$ be the subclass of functions with compact support in $B$. We define the Sobolev constant $C_{\mathcal{S}}(p, q)$ and the Poincaré constant $C_{\mathcal{P}}(p, q)$ respectively by

$$
\sup _{f \in \operatorname{Lip}_{0}(B)} \frac{\|f\|_{L^{q}(B)}}{|B|^{\frac{1}{n}+\frac{1}{q}-\frac{1}{p}}\|\nabla f\|_{L^{p}(B)}}=C_{\mathcal{S}}(p, q)
$$

and

$$
\sup _{f \in \operatorname{Lip}(B)} \frac{\left\|f-f_{a v}\right\|_{L^{q}(B)}}{|B|^{\frac{1}{n}+\frac{1}{q}-\frac{1}{p}}\|\nabla f\|_{L^{p}(B)}}=C_{\mathcal{P}}(p, q),
$$

where

$$
f_{a v}=\int_{B} f /|B|
$$

Both $C_{\mathcal{S}}(p, q)$ and $C_{\mathcal{P}}(p, q)$ are independent of $B$, as can be seen by translation and dilation.

Similarly, for a bounded domain $\Omega \subset \mathbb{R}^{n}$, we define corresponding constants $C_{\mathcal{S}}(p, q, \Omega)$ and $C_{\mathcal{P}}(p, q, \Omega)$ by replacing $B$ by $\Omega$ everywhere in the definitions above. By using symmetric decreasing rearrangements, it can be shown that $C_{\mathcal{S}}(p, q, \Omega) \leq$ $C_{\mathcal{S}}(p, q)$ for any $\Omega$. On the other hand, $C_{\mathcal{P}}(p, q, \Omega)$ is known to be finite only for reasonably nice domains $\Omega$, such as John domains and domains satisfying the cone condition; see for example [2], 24], [7], or [9].

Received by the editors March 3, 2005.

2000 Mathematics Subject Classification. Primary 26D10; Secondary 46 E35.

Key words and phrases. Poincaré inequalities, Sobolev inequalities, Hardy inequalities. 
In case $n>1$, the exact value of $C_{\mathcal{S}}(p, q)$ has been obtained for certain values of $p$ and $q$. For example, when $1 \leq p<n$ and $q=\frac{p n}{n-p}$,

$$
C_{\mathcal{S}}\left(p, \frac{p n}{n-p}\right)=\frac{1}{n \sqrt{\pi}}\left(\frac{n ! \Gamma(n / 2)}{2 \Gamma(n / p) \Gamma(n+1-n / p)}\right)^{1 / n}\left(\frac{n(p-1)}{n-p}\right)^{1-\frac{1}{p}} ;
$$

see [28], 4] or [22]. Moreover, in case $p=q=2$, the value is known to be closely related to the first eigenvalue of the Dirichlet problem

$$
\Delta u+\lambda u=0 \text { in } B ; u=0 \text { on } \partial B .
$$

In fact, if $B_{1}$ denotes the unit ball, then $C_{\mathcal{S}}(2,2)\left|B_{1}\right|^{1 / n}$ is the reciprocal of the first positive zero of the Bessel function $J_{n / 2-1}$; see for example [17. For any $p$ and $q$, it follows by using symmetric decreasing rearrangements that $C_{\mathcal{S}}(p, q) n^{\frac{1}{p}-\frac{1}{q}}\left|B_{1}\right|^{\frac{1}{n}}$ is the best constant $H_{p, q}$ in the inequality

$$
\left(\int_{0}^{1}|f(x)|^{q} x^{n-1} d x\right)^{1 / q} \leq H_{p, q}\left(\int_{0}^{1}\left|f^{\prime}(x)\right|^{p} x^{n-1} d x\right)^{1 / p}
$$

for all Lipschitz continuous functions on $[0,1]$ with $f(1)=0$. This observation is simple to prove, although we have been unable to locate any reference. In case either $p=1$ or $q=1$, Kufner and Opic [19] have obtained a sharp estimate for $H_{p, q}$ (see also [10, Proposition 1.7]). In fact, by putting all the weights in [19] equal to $x^{n-1}$, one finds that the best values are

$$
\begin{gathered}
H_{1, q}=n^{-1 / q}, \text { provided } 1 \leq q \leq n /(n-1), \\
H_{p, 1}=\left(\frac{1}{n+p^{\prime}}\right)^{1 / p^{\prime}} \frac{1}{n}, \text { for all } p>1 .
\end{gathered}
$$

One can then compute the corresponding values of $C_{\mathcal{S}}(p, q)$, namely,

$$
C_{\mathcal{S}}(1, q)=C_{\mathcal{S}}(1,1)=\frac{1}{n\left|B_{1}\right|^{1 / n}} \text { if } 1 \leq q \leq \frac{n}{n-1}
$$

(see also [15, (10.30), page 276] when $p=q=1$ ) and

$$
C_{\mathcal{S}}(p, 1)=\frac{1}{n^{1 / p}\left|B_{1}\right|^{1 / n}} \frac{1}{\left(n+p^{\prime}\right)^{1 / p^{\prime}}} \text { if } p>1
$$

Unfortunately, specific estimates related to $C_{\mathcal{P}}(p, q, \Omega)$ have been obtained only in very special cases, and then with different normalizations from the definition of $C_{\mathcal{S}}(p, q, \Omega)$. For example, in the case of convex domains $\Omega \subset \mathbb{R}^{n}$, it is shown in 25 ] and [5] that for $p=q=2$ and any fixed $n$,

$$
\sup _{\Omega \subset \mathbb{R}^{n}, \text { convex }} \sup _{f \in \operatorname{Lip}(\Omega)} \frac{\left\|f-f_{a v}\right\|_{L^{2}(\Omega)}}{(\operatorname{diam} \Omega)\|\nabla f\|_{L^{2}(\Omega)}}=\frac{1}{\pi}
$$

and it is shown in [1] that for $p=q=1$ and any fixed $n$,

$$
\sup _{\Omega \subset \mathbb{R}^{n}, \text { convex }} \sup _{f \in \operatorname{Lip}(\Omega)} \frac{\left\|f-f_{a v}\right\|_{L^{1}(\Omega)}}{(\operatorname{diam} \Omega)\|\nabla f\|_{L^{1}(\Omega)}}=\frac{1}{2} \text {. }
$$

The main technique used to prove these results is to decompose a convex domain (according to a given function $f$ ) into nonoverlapping arbitrarily thin domains (i.e., domains contained in arbitrarily thin rectangles), keeping the same average of $f$ on each subdomain, and then to apply a sharp weighted 1-dimensional 
Poincaré inequality to these subdomains with a weight which is a positive power of a nonnegative concave function. Moreover, it has recently been shown in [11] by a similar method that the best constant $C$ in the weighted Poincaré inequality for $1 \leq q \leq p<\infty$

$$
\left\|f-f_{a v}\right\|_{L_{w}^{q}(\Omega)} \leq C w(\Omega)^{\frac{1}{q}-\frac{1}{p}} \operatorname{diam}(\Omega)\|\nabla f\|_{L_{w}^{p}(\Omega)},
$$

where $\Omega, f$ and $w$ are allowed to range respectively over the classes of bounded convex domains in $\mathbb{R}^{n}$, Lipschitz continuous functions on $\Omega$, and weights which are any positive power of a nonnegative concave function on $\Omega$, is the same as the best constant for the corresponding 1-dimensional situation.

From now on, we will discuss just the 1-dimensional case of the above. Then $C_{\mathcal{P}}(p, q)$ is defined by

$$
\sup _{f \in \operatorname{Lip}[a, b]} \frac{\left\|f-f_{a v}\right\|_{L^{q}[a, b]}}{\left\|f^{\prime}\right\|_{L^{p}[a, b]}}=C_{\mathcal{P}}(p, q)(b-a)^{1+\frac{1}{q}-\frac{1}{p}},
$$

where $f_{a v}=\int_{a}^{b} f(x) d x /(b-a)$. Again, it follows by translation and dilation that $C_{\mathcal{P}}(p, q)$ is independent of $[a, b]$. Also, the Sobolev and Hardy constants $C_{\mathcal{S}}(p, q)$ and $C_{\mathcal{H}}(p, q)$ are defined by

$$
\begin{gathered}
\sup _{f \in \operatorname{Lip}_{0}[a, b]} \frac{\|f\|_{L^{q}[a, b]}}{\left\|f^{\prime}\right\|_{L^{p}[a, b]}}=C_{\mathcal{S}}(p, q)(b-a)^{1+\frac{1}{q}-\frac{1}{p}}, \\
\sup _{f \in \operatorname{Lip}[a, b]} \frac{\|f-f(a)\|_{L^{q}[a, b]}}{\left\|f^{\prime}\right\|_{L^{p}[a, b]}}=C_{\mathcal{H}}(p, q)(b-a)^{1+\frac{1}{q}-\frac{1}{p}} .
\end{gathered}
$$

The value of $C_{\mathcal{H}}(p, q)$ is unchanged if $f(a)$ is replaced by $f(b)$ in the definition; this follows as before by an appropriate affine change of variables. In the definition of $C_{\mathcal{H}}$, we may further restrict the functions $f$ by requiring that $f(a)=0$ since both $f$ and $f-f(a)$ have the same derivative.

The 1-dimensional constant $C_{\mathcal{S}}(p, q)$ is given in Talenti [28, page 357]:

$$
C_{\mathcal{S}}(p, q)=\frac{q\left(1+p^{\prime} / q\right)^{1 / p} \Gamma\left(\frac{1}{q}+\frac{1}{p^{\prime}}\right)}{2\left(1+q / p^{\prime}\right)^{1 / q} \Gamma(1 / q) \Gamma\left(1 / p^{\prime}\right)}
$$

for $1 \leq q<\infty$ and $1<p<\infty$.

Moreover, when $p=1$, it is shown in [19] that $C_{\mathcal{H}}(1, q)=1$, and when $q=1$ and $p>1$, it is a consequence of the results in [19] (by putting all the weights there equal to 1) that

$$
C_{\mathcal{H}}(p, 1)=\left(\frac{1}{1+p^{\prime}}\right)^{1 / p^{\prime}} ;
$$

see also [10, Proposition 1.6]. Furthermore, let us note that it is simple to see that $C_{\mathcal{S}}(p, q)=C_{\mathcal{H}}(p, q) / 2$; see Remarks $1.4(4)$.

On the other hand, not much is known about $C_{\mathcal{P}}(p, q)$ when $n=1$ except for certain values of $p$ and $q$. Recently, it was shown by the authors [10] that

$$
C_{\mathcal{P}}(1, q)=\max _{x \in[0,1]}\left(x^{q}(1-x)+(1-x)^{q} x\right)^{1 / q} .
$$

Note that the above constant equals $1 / 2$ for $1 \leq q \leq 3$ but strictly exceeds $1 / 2$ for $q>3$; see the Appendix in [10, and also [23] in case $q=1$. Moreover, it 
is easy to check that it equals $(1 / 12)^{1 / 4}$ when $q=4$. By considering a variation of the Dirichlet problem, it is shown in [25] that $C_{\mathcal{P}}(2,2)=1 / \pi$. Furthermore, $C_{\mathcal{P}}(1,1)=1 / 2$ as already mentioned. Note that $C_{\mathcal{P}}(2,2)$ and $C_{\mathcal{P}}(1,1)$ are the same as the constants given in (1.3) and (1.4) since (1.3) and (1.4) are independent of $n$.

We begin by deriving the following simple inequality relating $C_{\mathcal{P}}(p, q)$ and $C_{\mathcal{H}}(p, q)$.

Proposition 1.1. If $1 \leq p, q<\infty$, then

$$
C_{\mathcal{P}}(p, q) \geq \frac{1}{2} C_{\mathcal{H}}(p, q)
$$

Proof. Recall that $C_{\mathcal{P}}(p, q)$ and $C_{\mathcal{H}}(p, q)$ are independent of the interval $[a, b]$. Fix $[a, b]$, let $c=\frac{a+b}{2}$, and consider any $f$ which is Lipschitz continuous on $[c, b]$ with $f(c)=0$. Define $\tilde{f}$ on $[a, b]$ by $\tilde{f}=f$ on $[a, c]$ and $\tilde{f}(x)=-f(2 c-x)$ on $[c, b]$. Then

$$
\tilde{f}_{a v}=0,\|\tilde{f}\|_{L^{q}[a, b]}=2^{\frac{1}{q}}\|f\|_{L^{q}[c, b]},\left\|\tilde{f}^{\prime}\right\|_{L^{p}[c, b]}=2^{\frac{1}{p}}\left\|f^{\prime}\right\|_{L^{p}[a, c]},
$$

and therefore, since $b-a=2(b-c)$,

$$
C_{\mathcal{P}}(p, q)[2(b-c)]^{1+\frac{1}{q}-\frac{1}{p}} \geq \frac{\left\|\tilde{f}-\tilde{f}_{a v}\right\|_{L^{q}[a, b]}}{\left\|\tilde{f}^{\prime}\right\|_{L^{p}[a, b]}}=2^{\frac{1}{q}-\frac{1}{p}} \frac{\|f-f(c)\|_{L^{q}[c, b]}}{\left\|f^{\prime}\right\|_{L^{p}[c, b]}} .
$$

The proposition now follows by taking the supremum over $f$.

Note that the converse of the inequality in the proposition is not true in general; for example recall that $C_{\mathcal{H}}(1,4)=1$ while $C_{\mathcal{P}}(1,4)=(1 / 12)^{1 / 4}$.

In this note, we will show that the converse of the inequality in the proposition is true in case $q=1$ and $1<p<\infty$.

Theorem 1.2. Let $1<p<\infty$. Then $C_{\mathcal{P}}(p, 1)=\frac{1}{2} C_{\mathcal{H}}(p, 1)$. In particular,

$$
\sup _{f \in \operatorname{Lip}[a, b]} \frac{\left\|f-f_{a v}\right\|_{L^{1}[a, b]}}{\left\|f^{\prime}\right\|_{L^{p}[a, b]}}=\frac{1}{2}\left(\frac{1}{1+p^{\prime}}\right)^{1 / p^{\prime}}(b-a)^{2-\frac{1}{p}} .
$$

First, let us establish the following lemma. We say that $f$ is piecewise linear on $[a, b]$ if $f$ is continuous on $[a, b]$ and there exist points $a_{0}=a<a_{1}<a_{2}<\cdots<$ $a_{n}=b$ such that the graph of $f$ is a line segment on each $\left[a_{i-1}, a_{i}\right]$. We also define the nonincreasing rearrangement $f^{*}(t), t \in[0, b-a]$, of such an $f$ by

$$
f^{*}(t)=\inf \left\{y \geq f_{\min }: \omega_{f}(y) \leq t\right\},
$$

where $f_{\min }=\min \{f(x): x \in[a, b]\}$ and $\omega_{f}(y)$ is the distribution function of $f$, i.e., $\omega_{f}(y)$ is the Lebesgue measure of the set $\{x \in[a, b]: f(x)>y\}, y \geq f_{\min }$.

Lemma 1.3. If $f$ is a piecewise linear function on $[a, b]$, then for all $1 \leq p<\infty$, $\left\|f^{*}-f_{a v}^{*}\right\|_{L^{p}[0, b-a]}=\left\|f-f_{a v}\right\|_{L^{p}[a, b]}, \quad f_{a v}^{*}=f_{a v}$ and $\left\|\left(f^{*}\right)^{\prime}\right\|_{L^{p}[0, b-a]} \leq\left\|f^{\prime}\right\|_{L^{p}[a, b]}$, where $f^{*}$ is the nonincreasing rearrangement of $f$.

Proof. Let $f$ be a piecewise linear function on $[a, b]$. Then there exist $a=a_{0}<$ $a_{1}<a_{2}<\cdots<a_{n}=b$ such that $f$ is linear on each $\left[a_{i-1}, a_{i}\right]$. Let us arrange $\left\{f\left(a_{0}\right), \cdots, f\left(a_{n}\right)\right\}$ in nondecreasing order:

$$
\left\{f\left(\alpha_{0}\right), f\left(\alpha_{1}\right), \cdots, f\left(\alpha_{n}\right)\right\} .
$$


Note that $\left\{\alpha_{0}, \alpha_{1}, \cdots, \alpha_{n}\right\}$ is just a permutation of $\left\{a_{0}, a_{1}, \cdots, a_{n}\right\}$, and there exist $0=b_{0} \leq b_{1} \leq b_{2} \leq \cdots \leq b_{n}=b-a$ such that $\left[f\left(\alpha_{i-1}\right), f\left(\alpha_{i}\right)\right]=f^{*}\left[b_{i-1}, b_{i}\right]$ and $f^{*}$ is linear on $\left[b_{i-1}, b_{i}\right]$. Clearly,

$$
f^{-1}\left(f\left(\alpha_{i-1}\right), f\left(\alpha_{i}\right)\right) \cap f^{-1}\left(f\left(\alpha_{j-1}\right), f\left(\alpha_{j}\right)\right)=\emptyset \text { if } i \neq j .
$$

Moreover, for each $i$,

$$
f^{-1}\left(f\left(\alpha_{i-1}\right), f\left(\alpha_{i}\right)\right) \text { is either empty or a union } \bigcup_{j=1}^{k_{i}}\left(c_{j}, d_{j}\right)
$$

such that for each $j$, the graph of $f$ is a line segment on $\left[c_{j}, d_{j}\right]$ and $\max \left\{f\left(c_{j}\right), f\left(d_{j}\right)\right\}$ $=f^{*}\left(b_{i-1}\right), \min \left\{f\left(c_{j}\right), f\left(d_{j}\right)\right\}=f^{*}\left(b_{i}\right)$. Note that $\sum_{j}\left(d_{j}-c_{j}\right)=b_{i}-b_{i-1}$. It is clear that

$$
\begin{aligned}
\int_{b_{i-1}}^{b_{i}}\left|\left(f^{*}\right)^{\prime}\right|^{p} d x & =\left(b_{i}-b_{i-1}\right)^{1-p}\left|f^{*}\left(b_{i}\right)-f^{*}\left(b_{i-1}\right)\right|^{p} \\
& \leq\left(d_{j}-c_{j}\right)^{1-p}\left|f\left(d_{j}\right)-f\left(c_{j}\right)\right|^{p} \text { for all } j .
\end{aligned}
$$

Consequently,

$$
\int_{b_{i-1}}^{b_{i}}\left|\left(f^{*}\right)^{\prime}\right|^{p} d x \leq \sum_{j} \int_{c_{j}}^{d_{j}}\left|f^{\prime}\right|^{p} d x
$$

and

$$
\left\|\left(f^{*}\right)^{\prime}\right\|_{L^{p}[0, b-a]} \leq\left\|f^{\prime}\right\|_{L^{p}[a, b]} .
$$

Finally, since both $f$ and $f^{*}$ have the same distribution function, we have $f_{a v}^{*}=f_{a v}$ and

and the lemma follows.

$$
\left\|f^{*}-f_{a v}^{*}\right\|_{L^{p}[0, b-a]}=\left\|f-f_{a v}\right\|_{L^{p}[a, b]},
$$

Remarks 1.4. (1) Our proof of the lemma is a modification of a method of Hildén 18. However, our computation is simpler since piecewise linear functions in one dimension have very simple structure.

(2) The lemma remains true for all Lipshitz functions by using an approximation argument based on the density of piecewise linear functions as in [18] or [14]. The lemma also has an analogue for nondecreasing rearrangements.

(3) Let $f_{\max }=\max \{f(x): x \in[a, b]\}$. Since $f_{\max }=\left(f^{*}\right)_{\max }=f^{*}(0)$, it follows from the lemma and the density of piecewise linear functions in Sobolev spaces that

$$
\sup _{f \in \operatorname{Lip}[a, b]} \frac{\left\|f-f_{\text {max }}\right\|_{L^{q}[a, b]}}{\left\|f^{\prime}\right\|_{L^{p}[a, b]}}=C_{\mathcal{H}}(p, q)(b-a)^{1+\frac{1}{q}-\frac{1}{p}} .
$$

Moreover, the analogue of (1.8) with $f_{\max }$ replaced by $f_{\min }$ holds.

(4) Similar conclusions hold for the symmetric nonincreasing rearrangement of a nonnegative Lipschitz continuous function $f$ which vanishes outside $[a, b]$, and therefore

$$
\sup _{f \in \operatorname{Lip}_{0}[a, b]} \frac{\|f\|_{L^{q}[a, b]}}{\left\|f^{\prime}\right\|_{L^{p}[a, b]}}=\frac{1}{2} C_{\mathcal{H}}(p, q)(b-a)^{1+\frac{1}{q}-\frac{1}{p}},
$$

or $C_{\mathcal{H}}(p, q)=2 C_{\mathcal{S}}(p, q)$. Indeed, in the study of sharp constants for (1.1), symmetric nonincreasing rearrangements have been widely used; see for example [28].

We are now ready to prove the theorem. 
Proof of Theorem 1.2. Let $P W(a, b)$ denote the collection of piecewise linear functions on $[a, b]$. Using the fact that for any Lipschitz function $f$ on $[a, b]$ and $\varepsilon>0$, there is a piecewise linear function $f_{\varepsilon}$ on $[a, b]$ such that

$$
\left\|f-f_{\varepsilon}\right\|_{L^{1}[a, b]},\left\|f_{\varepsilon}^{\prime}-f^{\prime}\right\|_{L^{p}[a, b]}<\varepsilon,
$$

one has

$$
\sup _{f \in \operatorname{Lip}[a, b]} \frac{\left\|f-f_{a v}\right\|_{L^{1}[a, b]}}{\left\|f^{\prime}\right\|_{L^{p}[a, b]}}=\sup _{f \in P W(a, b)} \frac{\left\|f-f_{a v}\right\|_{L^{1}[a, b]}}{\left\|f^{\prime}\right\|_{L^{p}[a, b]}} .
$$

By translation and dilation, it is enough to prove the theorem for $a=0$ and $b=1$. Moreover, by the lemma, it suffices to consider just nonincreasing functions $f$. Since $f$ is monotone, there exists $\alpha \in(0,1)$ such that $f(\alpha)=f_{a v}$. We may also assume that $f_{a v}=0$, and therefore

$$
\|f\|_{L^{1}[0, \alpha]}=\|f\|_{L^{1}[\alpha, 1]} .
$$

We may assume without loss of generality that

$$
\alpha^{2-\frac{1}{p}}\left\|f^{\prime}\right\|_{L^{p}[0, \alpha]} \geq(1-\alpha)^{2-\frac{1}{p}}\left\|f^{\prime}\right\|_{L^{p}[\alpha, 1]} .
$$

Let us define a function $g$ on $[-1 / 2,1 / 2]$ by

$$
g(x)=\left\{\begin{array}{cc}
2(1-\alpha) f(2(1-\alpha) x+\alpha) & \text { if } x \in[0,1 / 2], \\
-2(1-\alpha) f(-2(1-\alpha) x+\alpha) & \text { if } x \in[-1 / 2,0] .
\end{array}\right.
$$

Note that $g(x)=-g(-x), g(0)=0$,

$$
\|g\|_{L^{1}[-1 / 2,1 / 2]}=2\|f\|_{L^{1}[\alpha, 1]}=\|f\|_{L^{1}[0,1]}
$$

and

$$
\left\|g^{\prime}\right\|_{L^{p}[-1 / 2,1 / 2]}^{p}=2(2(1-\alpha))^{2 p-1} \|\left. f^{\prime}\right|_{L^{p}[\alpha, 1]} ^{p} .
$$

Furthermore, by assumption,

$$
\left\|f^{\prime}\right\|_{L^{p}[0,1]}^{p} \geq\left\{\left(\frac{1-\alpha}{\alpha}\right)^{2 p-1}+1\right\}\left\|f^{\prime}\right\|_{L^{p}[\alpha, 1]}^{p} .
$$

Since for all $\alpha \in(0,1)$, we have

$$
\left(\frac{1}{1-\alpha}\right)^{2 p-1}+\left(\frac{1}{\alpha}\right)^{2 p-1} \geq 2^{2 p}
$$

it follows by combining estimates that

$$
\|f\|_{L^{1}[0,1]} /\left\|f^{\prime}\right\|_{L^{p}[0,1]} \leq\|g\|_{L^{1}[-1 / 2,1 / 2]} /\left\|g^{\prime}\right\|_{L^{p}[-1 / 2,1 / 2]} .
$$

Therefore,

$$
\begin{gathered}
C_{\mathcal{P}}(1, p)=\sup \left\{\frac{\|f\|_{L^{1}[-1 / 2,1 / 2]}}{\left\|f^{\prime}\right\|_{L^{p}[-1 / 2,1 / 2]}}: f \text { is monotone, } f(x)=-f(-x)\right\} \\
\leq 2^{1-\frac{1}{p}} \sup \left\{\frac{\|f\|_{L^{1}[0,1 / 2]}}{\left\|f^{\prime}\right\|_{L^{p}[0,1 / 2]}}: f(0)=0\right\}=2^{1-\frac{1}{p}} 2^{\frac{1}{p}-2} C_{\mathcal{P}}(1, p)=\frac{1}{2} C_{\mathcal{H}}(1, p) .
\end{gathered}
$$

Thus the converse of the inequality in the proposition holds, and the theorem is proved. 
Final remarks. (1) There exists a Lipschitz continuous function on $[-1 / 2,1 / 2]$ such that the best constant is achieved. In fact, let $f$ be the odd function on $[-1 / 2,1 / 2]$ with

Then $f_{a v}=0$ and

$$
f(x)=\int_{0}^{x}\left(\frac{1}{2}-t\right)^{p^{\prime} / p} d t \text { on }[0,1 / 2]
$$

$$
\|f\|_{L^{1}[-1 / 2,1 / 2]}=\frac{1}{2\left(1+p^{\prime}\right)^{1 / p^{\prime}}}\left\|f^{\prime}\right\|_{L^{p}[-1 / 2,1 / 2]} .
$$

(2) For other values of $p, q$, bounds for $C_{\mathcal{H}}(p, q)$ have been obtained in [19]. In case $1 \leq p \leq q<\infty$, bounds for $C_{\mathcal{P}}(p, q)$ have been obtained in 10. However, only upper bounds are obtained in [10] in case $1 \leq q<p<\infty$.

(3) Since Lipschitz continuous functions are dense in the Sobolev space $W^{1, p}[a, b]$, it is obvious that the following inequality is sharp:

$$
\left\|f-f_{a v}\right\|_{L^{1}[a, b]} \leq \frac{1}{2\left(1+p^{\prime}\right)^{1 / p^{\prime}}}(b-a)^{2-\frac{1}{p}}\left\|f^{\prime}\right\|_{L^{p}[a, b]}
$$

for all $f \in W^{1, p}[a, b]$.

Added note. We would like to thank G. Talenti for alerting us to the work of A. Cianchi 12 concerning the constant $C_{\mathcal{P}}(p, q)$ in (1.2). In case $p=1$ and $0<q \leq n /(n-1), n \geq 2$, it is shown in [12] that if $V_{n}=\frac{2 \pi^{n / 2}}{n \Gamma(n / 2)}$ (the volume of the $n$-dimensional unit ball) and $r$ is the radius of the ball $B$, then

$$
C_{\mathcal{P}}(1, q)|B|^{\frac{1}{n}+\frac{1}{q}-1}=\frac{V_{n}^{1 / q} r^{\frac{n}{q}-n+1}}{2 V_{n-1}}=\frac{1}{2}|B|^{\frac{1}{n}+\frac{1}{q}-1} \frac{V_{n}^{(n-1) / n}}{V_{n-1}} .
$$

Thus, for $n \geq 2$ and $0<q \leq n /(n-1)$,

$$
C_{\mathcal{P}}(1, q)=\frac{1}{2} \frac{V_{n}^{(n-1) / n}}{V_{n-1}}
$$

\section{REFERENCES}

1. G. Acosta and R. G. Durán, An optimal Poincaré inequality in $L^{1}$ for convex domains, Proc. Amer. Math. Soc. 132 (2004), 195-202. MR2021262 (2004j:26031)

2. R. Adams, Sobolev Spaces, Academic Press, New York, 1975. MR0450957 (56:9247)

3. F. J. Almgren and E. H. Lieb, Symmetric rearrangement is sometimes continuous, J. Amer. Math. Soc. 2 (1989), 683-773. MR1002633 (90f:49038)

4. T. Aubin, Problèmes isopérimétriques et espaces de Sobolev, J. Diff. Geometry 11 (1976), 573-598. MR0448404 (56:6711)

5. M. Bebendorf, A note on Poincaré's inequality for convex domains, Z. Anal. Anwendungen 22 (2003), 751-756. MR2036927 (2004k:26025)

6. W. Beckner and M. Pearson, On sharp Sobolev embedding and the logarithmic Sobolev inequality, Bull. London Math Soc. 30 (1998), 80-84. MR1479040 (98k:46048)

7. B. Bojarski, Remarks on Sobolev imbedding inequalities, Complex Analysis, Lecture Notes in Math., 1351, Springer-Verlag, 1989, 52-68. MR0982072 (90b:46068)

8. S.-K. Chua, Extension theorems on weighted Sobolev spaces, Indiana Math. J. 41 (1992), no. 4, 1027-1076. MR1206339 (94a:46035)

9. S.-K. Chua, Weighted Sobolev's inequalities on domains satisfying the chain condition, Proc. Amer. Math. Soc. 117 (1993), no. 2, 449-457. MR1140667 (93d:46050)

10. S.-K. Chua and R. L. Wheeden, Sharp conditions for weighted 1-dimensional Poincaré inequalities, Indiana Math. J. 49 (2000), 143-175. MR.1777034 (2001h:26021)

11. S.-K. Chua and R. L. Wheeden, Estimates of best constants for weighted Poincaré inequalities on convex domains, Proc. London Math Soc., to appear. 
12. A. Cianchi, A sharp form of Poincaré type inequalities on balls and spheres, Z. Angew. Math. Phys. 40 (1989), 558-569. MR1008923 (90k:49040)

13. A. Cianchi, D. E. Edmunds and P. Gurka, On weighted Poincaré inequalities, Math. Nachr. 180 (1996), 15-41. MR.1397667 (97e:26015)

14. J.-M. Coron, The continuity of rearrangement in $W^{1, p}(\mathbb{R})$, Ann. Scuola Norm. Sup. Pisa Cl. Sci. (4) 11 (1984), 57-85. MR0752580 (86a:46035)

15. D. Gilbarg and N. S. Trudinger, Elliptic Partial Differential Equations of Second Order, Springer-Verlag, 1997. MR1814364 (2001k:35004)

16. P. Gurka and A. Kufner, A note on a two-weighted Sobolev inequality, Approximation and Function Spaces, 169-172, Banach Center Publ. 22, Warsaw, 1989. MR1097190 (92b:46040)

17. W. Hayman, Some bounds for principal frequency, Applicable Analysis 7 (1978), 247-254. MR0492339 (58:11468)

18. K. Hildén, Symmetrization of functions in Sobolev spaces and the isopermetric inequality, Manuscripta Math. 18 (1976), 215-235. MR0409773 (53:13525)

19. A. Kufner and B. Opic, Hardy-type Inequalities, Pitman Research Notes 219, Longman House, U. K., 1990. MR.1069756 (92b:26028)

20. E. H. Lieb, Gaussian kernels have only Gaussian maximizers, Invent. Math. 102 (1990), 179208. MR1069246 (91i:42014)

21. E. H. Lieb, Sharp constants in the Hardy-Littlewood-Sobolev and related inequalities, Ann. of Math. 118 (1983), 349-374. MR0717827 (86i:42010)

22. P. L. Lions, The concentration-compactness principle in calculus of variations, The limit case, Part I \& II, Rev. Mat. Iberoamericana 1 (1985), 145-201; 2 (1985), 45-121. MR0850686 $(87 \mathrm{j}: 49012)$

23. G. Lu and R. L. Wheeden, Poincaré inequalities, isoperimetric estimates and representation formulas on product spaces, Indiana Math. J. 47 (1998), 123-151. MR1631545 (99e:46043)

24. V. G. Maz'ja, Sobolev Spaces, Springer-Verlag, New York, 1985. MR0817985 (87g:46056)

25. L. E. Payne and H. F. Weinberger, An optimal Poincaré inequality for convex domains, Arch. Rat. Mech. Anal. 5 (1960), 286-292. MR0117419 (22:8198)

26. G. Rosen, Minimum value for $c$ in the Sobolev inequality $\|\phi\|_{6} \leq c\|\nabla \phi\|_{2}$, Siam. J. Appl. Math. 21 (1971), 30-32. MR0289739 (44:6927)

27. G. Sinnamon, Weighted Hardy and Opial type inequalities, J. Math. Anal. Appl. 160 (1991), 434-445. MR 1126128 (92f:26037)

28. G. Talenti, Best constant in Sobolev inequality, Ann. Mat. Pura Appl. 110 (1976), 353-372. MR.0463908 (57:3846)

29. G. Talenti, Some inequalities of Sobolev type on two-dimensional spheres, General Inequalities 5, 5th International Conference on General Inequalities, Oberwolfach (1986), 401-408. MR.1018163(90k:26031)

Department of Mathematics, National University of Singapore, 2 Science Drive 2, SiNGAPORE 117543

E-mail address: matcsk@nus.edu.sg

Department of Mathematics, Rutgers University, New Brunswick, New Jersey 08903

E-mail address: wheeden@math.rutgers.edu 